\title{
Analysis of LP Relaxations for Multiway and Multicut Problems
}

\author{
Dimitris Bertsimas, ${ }^{1}$ Chung-Piaw Teo, ${ }^{2}$ Rakesh Vohra ${ }^{3}$ \\ ${ }^{1}$ Sloan School of Management and Operations Research Center, \\ Massachusetts Institute of Technology, E53-363, Cambridge, Massachusetts 02139 \\ 2 Department of Decision Sciences, Faculty of Business Administration, \\ National University of Singapore, Singapore \\ ${ }^{3}$ School of Management, Northwestern University, Evanston, Illinois
}

Received 3 September 1996; accepted 23 October 1998

\begin{abstract}
We introduce in this paper an exact nonlinear formulation of the multiway cut problem. By simple linearizations of this formulation, we derive several well-known and new formulations for the problem. We further establish a connection between the multiway cut and the maximum-weighted independent set problem. This leads to the study of several instances of the multiway cut problem through the theory of perfect graphs. We also introduce a new randomized rounding argument to study the sharpness of these formulations. (C) 1999 John Wiley \& Sons, Inc. Networks 34: 102-114, 1999
\end{abstract}

Keywords: multicut problems; LP relaxation; randomized rounding

\section{INTRODUCTION}

Given a graph $G=(V, E)$ with edge weight $c_{e}$ for each $e$ $\in E$ and a set of terminal nodes $T=\left\{v_{1}, v_{2}, \ldots, v_{k}\right\}$ $\subset V$, a multiway cut is a set of edges whose removal disconnects every pair of terminal nodes. Equivalently, this gives rise to a partition of the node set into $k$ or more classes, each containing exactly one or none of the terminal

A preliminary version of this article appears under the title "Nonlinear formulations and improved randomized approximation algorithms for multiway and multicut problems," E. Balas and J. Clausen (Editors), Proc 4th Integer Programming and Combinatorial Optimization Conference, LNCS 920, 29-39, Springer-Verlag, Berlin, 1995, pp. 29-39.

Correspondence to: D. Bertsimas

Contract grant sponsor: Draper Laboratory

Contract grant sponsor: NSF; contract grant number: DMI-9610486 nodes. The edges between nodes in distinct classes will correspond to the edges in the multiway cut solution. The problem of finding the multiway cut of minimum total weight is called the multiway cut problem. When $T$ consists of only two terminals $(k=2)$, the problem is the wellknown minimum-cut problem. For $k \geq 3$, it was shown by Dalhaus et al. [8] to be NP-hard even on planar graphs.

The case $k=2$ is not the only polynomially solvable instance of the multiway cut problem. Lovász [13] and Cherkasskij [4] show that when $c_{e}=1 \forall e \in E$ and $G$ is Eulerian then the multiway cut problem is polynomially solvable. Erdos and Székely [9] showed that a generalization of the multiway cut problem is polynomially solvable when the underlying graph $G$ is a tree. Dalhaus et al. [8] showed the problem to be polynomially solvable for fixed $k$ on planar graphs. 
Chopra and Rao [6] and Cunningham [7] investigated the multiway cut problem using a polyhedral approach. They derived several valid inequalities and facets. For one particular formulation of the problem, Cunningham [7] showed that the value of the minimum multiway cut is at most twice the value of its linear relaxation. Chopra and Owen [5] proposed an extended formulation of the problem which was shown to be tighter than all previously proposed. In addition, when the underlying graph is a tree, they showed that the extended formulation is integral. They did not, however, analyze the tightness of their extended formulation, but relied on computational results to show that their formulation consistently yields high-quality solutions to the multiway cut problem.

Regarding approximation algorithms, Dalhaus et al. [8] proposed an $2(1-1 / k)$ approximation algorithm. Their approach can also be used to yield slightly improved bounds for the four-way and eight-way cut problems.

A more general problem that we also consider in the present paper is the multicut problem. Given a graph $G$ $=(V, E)$ with edge weights $c_{e}$ for each $e \in E$, and a demand graph $H=(V(H), E(H))$, find a minimum weight set of edges whose removal disconnects each node $s$ $\in V(H)$ from $t \in V(H)$ if $(s, t) \in E(H)$. If $V(H)$ is a complete graph on $k$ nodes, the multicut problem reduces to the multiway cut problem. Regarding approximation algorithms, Garg et al. [10] proposed an algorithm that produces a multicut whose weight is within $O(\log (|V(H)|))$ from the optimal solution.

Our overall goal in this paper is to study theoretically, using a new randomized rounding argument, the closeness of the value of the optimal multiway cut and multicut to the optimal solution value of the formulations proposed in Chopra and Owen [5]. Furthermore, we show that insights from the combinatorial approach can be used to sharpen the LP relaxations for the corresponding problems, using a simple rounding argument of the underlying fractional solutions. The randomized rounding approach proposed in this paper has also found applications in several other problems; see, for instance, Bertsimas et al. [3] and Teo and Bertsimas [15].

Our contributions and the structure of the paper are as follows:

1. In Section 2, we express the multiway cut problem as a continuous nonlinear program. The formulation is exact in the sense that its optimal solution is integral. The fact that a $0-1$ integer program can be expressed exactly as a compact nonlinear program is not surprising. If $x$ is a $0-1$ variable, this can be expressed as $x(1-x)=0$. What is of interest is that the constraints of our formulation are linear. The nonlinear formulation provides a framework for the study of extended formulations. Many of the known standard and extended formulations and valid inequalities can be derived from simple lineariza- tions of the nonlinear formulation. This provides a systematic way to construct improved extended formulations for the multiway cut problem. In particular, we derive the extended formulations of Chopra and Owen [5].

2. In Section 3, we establish a connection between the multiway cut problem and the independent set problem. This allows us to derive relaxations for the multiway cut problem that are stronger than previously known. In addition, we use the theory of perfect graphs to prove the integrality of some extended formulations of the multiway cut problem that have a special structure. In this way, we identify new polynomially solvable cases. In particular, we obtain the integrality result of Chopra and Owen [5] mentioned above and the result of Erdos and Székely [9] as a corollary.

3. In Section 4, we analyze the tightness of the proposed LP relaxations for the multiway cut problem by probabilistically rounding the optimal fractional solution of the associated linear programs. If $Z_{L P}$ denotes the optimal objective function value of the linear relaxation, and $Z_{I P}$, the value of the optimal integer solution, we show that $Z_{I P} \leq 2(1-1 / k) Z_{L P}$.

4. In Section 5, we show how the techniques developed for the multiway cut problem apply to the more general multicut problem. The technique of Dalhaus et al. [8] can be used to design a $2 \alpha(H)(1-1 / k)$ approximation algorithm for the problem. Notice that the bound is a direct generalization of the bound for the multiway cut problem. In addition, it is different from the $O(\log (|E(H)|))$ bound derived by Garg et al. [10]. We exhibit in this section a natural formulation that attains the same bound.

\section{AN EXACT NONLINEAR FORMULATION AND ITS LINEARIZATIONS}

In this section, we present a continuous nonlinear formulation for the multiway cut problem, prove its validity through randomization, and linearize it to obtain tighter linear relaxations.

Let $T=\left\{v_{1}, v_{2}, \ldots, v_{k}\right\}$ denote the set of terminal nodes. Let $y^{j}(u)$ denote the decision variable that node $u$ belongs to the same component as $v_{j}$ in a multiway cut. Since the edge $(u, v)$ belongs to the multiway cut if and only if $y^{j}(u)$ and $y^{j}(v)$ are distinct for some $j$, the following is clearly a valid nonlinear formulation of the multiway cut problem:

$$
(N F) Z_{N F}=\min \sum_{(u, v) \in E} c(u, v)\left(1-\sum_{j=1}^{k} y^{j}(u) y^{j}(v)\right)
$$




$$
\begin{aligned}
& \text { subject to } \quad \sum_{j=1}^{k} y^{j}(u)=1 ; \quad \forall u \in V \backslash T \\
& y^{j}\left(v_{j}\right)=1 ; \quad \forall j=1, \ldots, k, v_{j} \in T \\
& y^{j}\left(v_{l}\right)=0 ; \quad \forall l \neq j, 1 \leq l, \\
& j \leq k, v_{l} \in T \\
& y^{j}(u) \leq 1 ; \quad \forall u, v \in V \\
& y^{j}(u) \geq 0 ; \quad \forall u, v \in V \\
& y^{j}(u) \in\{0,1\} ; \quad \forall u, v \in V .
\end{aligned}
$$

Let $x(u, v)=1-\sum_{j=1}^{k} y^{j}(u) y^{j}(v), \forall(u, v) \in E$. Let $I Z_{M C}$ denote the value of a minimum multiway cut. It is clear that $I Z_{M C}=Z_{N F}$. Let $(N L F)$ denote the corresponding continuous relaxation of $(N F)$ [i.e., by removing the integrality constraint (6)]. Let $Z_{N L F}$ denote the corresponding optimal value. The following result shows that the continuous relaxation obtained in this way does not lead to any deterioration of the optimal solution value:

Theorem 1. $I Z_{M C}=Z_{N L F}$.

Proof. Let $(\bar{x}, \bar{y})$ be an optimal solution to Problem $(N L F)$. Node $u$ is assigned to the component of $v_{j}$ with probability $\bar{y}^{j}(u)$. Let $x$ be the incidence vector of the multiway cut obtained. Clearly,

$$
P\{\text { edge }(u, v) \text { in the multiway cut }\}=1-\sum_{j=1}^{k} \bar{y}^{j}(u) \bar{y}^{j}(v) \text {. }
$$

Hence,

$$
\begin{aligned}
E[x(u, v)]=P\{x(u, v)= & 1\} \\
& =1-\sum_{j=1}^{k} \bar{y}^{j}(u) \bar{y}^{j}(v)=\bar{x}(u, v),
\end{aligned}
$$

so $E\left[\sum_{(u, v) \in E} c(u, v) x(u, v)\right]=\sum_{(u, v) \in E} c(u, v) \bar{x}(u, v)$ $=Z_{N L F}$. The random process always produces a multiway cut solution, so its expected value cannot be smaller than the minimum. Hence, $I Z_{M C} \leq E\left[\sum_{(u, v) \in E} c(u, v) x(u, v)\right]$ $=Z_{N L F}$. Since all multiway cuts are feasible in $(N L F)$, $Z_{N L F} \leq I Z_{M C}$. Therefore, $I Z_{M C}=Z_{N L F}$.

Linearizing the previous formulation, we immediately obtain the extended formulation proposed by Chopra and Owen [5] using different considerations. In particular, since

$$
y^{j}(u) y^{j}(v) \leq \min \left(y^{j}(u), y^{j}(v)\right),
$$

by using the following inequality:

$$
\begin{aligned}
\sum_{j=1}^{k} y^{j}(u) y^{j}(v) \leq \sum_{j \in S} y^{j}(u) & \\
& +\sum_{j \notin S} y^{j}(v), \quad \forall S \subset\{1,2, \ldots, k\},
\end{aligned}
$$

we obtain the following extended formulation (relaxation) of the multiway cut problem:

$$
Z_{E F 1}=\min \sum_{(u, v) \in E} c(u, v) x(u, v)
$$

subject to

$$
\begin{aligned}
& x(u, v)+\sum_{j \in S} y^{j}(u)+\sum_{j \notin S} y^{j}(v) \geq 1, \\
& \forall S \subset\{1,2, \ldots, k\},(u, v) \in E
\end{aligned}
$$

and constraints (1)-(5). Here, the edge variables, $x(u, v)$, are considered the "natural" variables, while the node variables, $y^{j}(u)$, are viewed as the auxiliary variables.

Even though $(E F 1)$ has an exponential number of constraints, its linear relaxation can be solved in polynomial time. This is because the associated separation problem is solvable in polynomial time (see [5]). If we represent the product terms in $(N L F)$ by a variable:

$$
z^{j}(u, v)=y^{j}(u) y^{j}(v)
$$

we obtain a second extended formulation:

$$
Z_{E F 2}=\min \sum_{(u, v) \in E} c(u, v) x(u, v)
$$

subject to

$$
\begin{gathered}
x(u, v)+\sum_{j} z^{j}(u, v)=1, \\
\forall(u, v) \in E \\
z^{j}(u, v) \leq y^{j}(u), \quad \forall j,(u, v) \in E \\
z^{j}(u, v) \leq y^{j}(v), \quad \forall j,(u, v) \in E
\end{gathered}
$$$$
z^{j}(u, v) \geq y^{j}(u)+y^{j}(v)-1 \quad \forall j,(u, v) \in E
$$

and constraints (1)-(5). Under the condition that the weight function $c$ is nonnegative, it is easy to see that the constraints $z^{j}(u, v) \geq y^{j}(u)+y^{j}(v)-1$ are redundant. Chopra and Owen [5] proved that 
Theorem 2 [5]. When the cost function $c$ is nonnegative, $Z_{E F 1}=Z_{E F 2}$.

In contrast to $(E F 1)$, the formulation $(E F 2)$ involves only polynomially many variables and constraints. In the sequel, we will assume that $c_{e} \geq 0$ for all $e \in E$ and so we do not distinguish between $(E F 1)$ and $(E F 2)$.

Now, we derive a third extended formulation, equivalent to $(E F 2)$, not previously considered. This formulation will be used in the sequel in the analysis of the tightness of the formulations considered in this section.

Since $\sum_{j=1}^{k} y^{j}(u)=1$ in $(N L F)$, we can write

$$
x(u, v)=\sum_{j=1}^{k} y^{j}(u)-\sum_{j=1}^{k} y^{j}(u) y^{j}(v) .
$$

Replacing $u$ by $v$, we get

$$
x(u, v)=\sum_{j=1}^{k} y^{j}(v)-\sum_{j=1}^{k} y^{j}(u) y^{j}(v) .
$$

Adding these two equations together yields

$$
2 x(u, v)=\sum_{j=1}^{k}\left[y^{j}(u)+y^{j}(v)-2 y^{j}(u) y^{j}(v)\right] .
$$

Now,

$$
y^{j}(u)+y^{j}(v)-2 y^{j}(u) y^{j}(v) \geq\left|y^{j}(u)-y^{j}(v)\right|
$$

as long as $y^{j}(u), y^{j}(v) \leq 1$. Thus, we get the following convex programming formulation:

$$
Z_{E F 3}=\min \sum_{(u, v) \in E} c(u, v) x(u, v)
$$

subject to $2 x(u, v) \geq \sum_{j=1}^{k}\left|y^{j}(u)-y^{j}(v)\right|$,

$$
\forall(u, v) \in E
$$

and constraints (1)-(5). (EF3) can be turned into a linear program using the usual technique of introducing extra variables [i.e., replace $\left|y^{j}(u)-y^{j}(v)\right|$ by $w^{j}(u, v)$ and add the constraints $w^{j}(u, v) \geq y^{j}(u)-y^{j}(v), w^{j}(u, v) \geq y^{j}(v)$ $\left.-y^{j}(u)\right]$.

Theorem 3. When the cost function $c$ is nonnegative, $Z_{E F 2}$ $=Z_{E F 3}$.
Proof. Let $(\bar{x}, \bar{y}, \bar{z})$ be an optimal solution to $(E F 2)$. Pick any $u, v \in V$. Notice that $\bar{z}^{j}(u, v)=\min \left\{\bar{y}^{j}(u)\right.$, $\left.\bar{y}^{j}(v)\right\}$. Let $A=\left\{j: \bar{y}^{j}(u) \geq \bar{y}^{j}(v)\right\}$ and $A^{c}$ be the complement. Now, $(\bar{x}, \bar{y}, \bar{z})$ must satisfy

$$
\bar{x}(u, v)+\sum_{j} \bar{z}^{j}(u, v)=1=\sum_{j} \bar{y}^{j}(u)
$$

and

$$
\bar{x}(u, v)+\sum_{j} \bar{z}^{j}(u, v)=1=\sum_{j} \bar{y}^{j}(v) .
$$

Adding them together we get

$$
2 \bar{x}(u, v)+2 \sum_{j} \bar{z}^{j}(u, v)=\sum_{j} \bar{y}^{j}(u)+\sum_{j} \bar{y}^{j}(v) .
$$

Hence,

$$
2 \bar{x}(u, v)=\sum_{j}\left[\bar{y}^{j}(u)-\bar{z}^{j}(u, v)\right]+\sum_{j}\left[\bar{y}^{j}(v)-\bar{z}^{j}(u, v)\right] .
$$

If $j \in A$, then $\left|y^{j}(u)-y^{j}(v)\right|=y^{j}(u)-\bar{z}^{j}(u, v)$; otherwise, $\left|y^{j}(u)-y^{j}(v)\right|=y^{j}(v)-\bar{z}^{j}(u, v)$. Hence,

$$
\begin{array}{r}
2 \bar{x}(u, v)=\sum_{j \in A}\left[\bar{y}^{j}(u)-\bar{z}^{j}(u, v)\right]+\sum_{j \in A^{c}}\left[\bar{y}^{j}(v)-\bar{z}^{j}(u, v)\right] \\
=\sum_{j}\left|\bar{y}^{j}(u)-\bar{y}^{j}(v)\right| .
\end{array}
$$

Any optimal solution to $(E F 2)$ is thus a feasible solution to (EF3).

Now suppose that $(\bar{x}, \bar{y})$ is an optimal solution to $(E F 3)$. Pick any $u, v \in V$. Define $\bar{z}^{j}(u, v)=\min \left\{\bar{y}^{j}(u), \bar{y}^{j}(v)\right\}$. We show that $(\bar{x}, \bar{y}, \bar{z})$ is feasible for $(E F 2)$.

Let $A=\left\{j: \bar{y}^{j}(u) \geq \bar{y}^{j}(v)\right\}$ and $A^{c}$ be the complement. Then,

$$
\begin{aligned}
& \sum_{j}\left|\bar{y}^{j}(u)-\bar{y}^{j}(v)\right| \\
&=\sum_{j \in A}\left[\bar{y}^{j}(u)-\bar{y}^{j}(v)\right]+\sum_{j \in A^{c}}\left[\bar{y}^{j}(v)-\bar{y}^{j}(u)\right] .
\end{aligned}
$$

Also,

$$
2 \sum_{j} \bar{z}^{j}(u, v)=2 \sum_{j \in A} \bar{y}^{j}(v)+2 \sum_{j \in A^{c}} \bar{y}^{j}(u) .
$$

Since $2 \bar{x}(u, v)=\sum_{j}\left|\bar{y}^{j}(u)-\bar{y}^{j}(v)\right|$, it follows that 


$$
\begin{aligned}
2 \bar{x}(u, v)+2 \sum_{j} \bar{z}^{j}(u, v) & =\sum_{j \in A}\left[\bar{y}^{j}(u)-\bar{y}^{j}(v)+2 \bar{y}^{j}(v)\right] \\
& +\sum_{j \in A^{c}}\left[\bar{y}^{j}(v)-\bar{y}^{j}(u)+2 \bar{y}^{j}(u)\right]=2 .
\end{aligned}
$$

So, an optimal solution to $(E F 3)$ is feasible for $(E F 2)$.

By projecting out the auxiliary variables in these extended formulations, we can derive the standard formulations involving edge variables alone as well as several new valid inequalities for the multiway cut. This is described in the next theorem.

Theorem 4. Let L denote a subgraph of $G$ which contains some terminal nodes $v \in T$, which we label as $v_{1}, \ldots, v_{p}$, and at least one nonterminal node $w \in V T$. Suppose that the edges of $L$ can be oriented in such a way that there are exactly $q$ internally node disjoint paths from each $v_{j}$ to $w$, that $i s$, for all $j=1, \ldots, p$, all the paths from $v_{j}$ to $w$ are node-disjoint except for the nodes $v_{j}$ and $w$. Then,

$$
\sum_{e \in E(L)} x(e) \geq q(p-1)
$$

is a valid inequality for the multiway cut problem which is contained in the projection of (EF1) onto the space of the $x$ variables.

Proof. It is not difficult to show that the above inequality is valid. We prove a stronger result in that it is actually contained in the projection of (EF1). Consider an orientation of the edges of $L$ so that there are $q$ (fixed) internally disjoint directed paths from each terminal node $v_{j}$ to $w$. For each edge $(u, v)$ oriented from $u$ to $v$, let $S(u, v)$ denote the set of terminal nodes which use this edge along one of their $q$ paths to $w$. We have, from (EF1),

$$
x(u, v) \geq 1-\sum_{v_{j} \in S(u, v)} y^{j}(v)-\sum_{v_{j} \notin S(u, v)} y^{j}(u) .
$$

Note that if $v \neq w$ then $S\left(u_{1}, v\right), S\left(u_{2}, v\right)$ are disjoint, since the $q$ paths from each terminal node to $w$ are internally disjoint. Let $N^{+}(v), N^{-}(v)$ denote, respectively, the set of in-neighbors and out-neighbors of $v$ under the orientation. Let $u \in N^{-}(v)$. Note that the set $\{j: j \in S(v, u)\}$ is contained in $\cup\left\{j: j \in S(r, v), r \in N^{+}(v)\right\}$. Since $\Sigma_{j}$ $y^{j}(v)=1$, and

$$
\cup_{u \in N^{-}(v)} S(v, u)=\cup_{r \in N^{+}(v)} S(r, v),
$$

we have

$$
\begin{aligned}
\sum_{r \in N^{+}(v)} \sum_{j \in S(r, v)} y^{j}(v) & =\sum_{u \in N^{-}(v)} \sum_{j \in S(u, v)} y^{j}(v) \\
& =\sum_{u \in N^{-}(v)}\left(1-\sum_{j \notin S(v, u)} y^{j}(v)\right) .
\end{aligned}
$$

Hence, we have

$$
-\sum_{r \in N^{+}(v)} \sum_{j \in S(r, v)} y^{j}(v)+\sum_{u \in N^{-}(v)}\left\{1-\sum_{j \notin S(v, u)} y^{j}(v)\right\}=0 .
$$

On the other hand, each terminal node has degree at least $q$, and for each neighbor $u$ of a terminal node,

$$
x\left(v_{j}, u\right) \geq 1-y^{j}(u) .
$$

So,

$$
\sum_{u \in N^{-}\left(v_{j}\right)} x\left(v_{j}, u\right) \geq q-\sum_{u \in N^{-}(v)} y^{j}(u)
$$

For the node $w$, since $w$ has no out-neighbor, and each terminal has exactly $q$ internally disjoint paths to $w$,

$$
\sum_{r \in N^{+}(w)} \sum_{j \in S(r, w)} y^{j}(w)=q \sum_{j} y^{j}(w)=q .
$$

By (13),

$$
\begin{aligned}
\sum_{e=(u, v) \in E(L)} x(u, v) & \\
\geq & \sum_{e=(u, v) \in E(L)}\left\{1-\sum_{j \in S(u, v)} y^{j}(v)-\sum_{j \notin S(u, v)} y^{j}(u)\right\} \\
= & q p+\sum_{v \neq v_{j}, v \neq w}\left(-\sum_{r \in N^{+}(v)} \sum_{j \in S(r, v)} y^{j}(v)\right. \\
& \left.+\sum_{u \in N^{-}(v)}\left\{1-\sum_{j \notin S(v, u)} y^{j}(v)\right\}\right) \\
& -\sum_{r \in N^{+}(w)} \sum_{j \in S(r, w)} y^{j}(w) \geq q p-q .
\end{aligned}
$$

Hence, the result follows.

As an example, pick any two nodes $v_{r}$ and $v_{s}$ in $T$ and let $L$ be any path of length not less than 1 between them. The theorem implies that

$$
\sum_{e \in L} x_{e} \geq 1
$$

If we apply the theorem to every path between every pair of terminal nodes, we get the path formulation of the multiway 
cut problem. To describe this formulation, let $P(i, j)$ be the set of paths between terminals $v_{i}$ and $v_{j}$ :

$$
\begin{gathered}
(P F) \quad Z_{P F}=\min \sum_{e \in E} c_{e} x_{e} \\
\text { s.t. } \sum_{e \in p} x_{e} \geq 1 ; \quad \forall p \in P(i, j) \forall v_{i}, v_{j} \in T \\
0 \leq x_{e} \leq 1 .
\end{gathered}
$$

As another example, pick a tree $S$ on $G$ all of whose leaves are in $T$ and no terminal node is a nonleaf node. Call such a tree a $T$-tree. Then, by the theorem, we get

$$
\sum_{e \in E(S)} x_{e} \geq|S \cap T|-1
$$

which are called the tree inequalities. Generating all tree inequalities gives us another formulation called the tree formulation that was considered by Chopra and Rao [6] and Cunningham [7]. To describe this formulation, let $\mathcal{T}$ be the set of all $T$-trees:

$$
\begin{aligned}
& \text { (TF) } Z_{T F}=\min \sum_{e \in E} c_{e} x_{e} \\
& \text { s.t. } \quad \sum_{e \in E(S)} x_{e} \geq|S \cap T|-1 ; \quad S \in T \\
& \quad 0 \leq x_{e} \leq 1 .
\end{aligned}
$$

In the same way, one can derive the odd-wheel inequalities and bipartite inequalities. These are known to be facets of the multiway cut polytope. (See Chopra and Rao [6]).

\subsection{Fractional Extreme Points}

The extended formulations considered above are rather powerful as their projections contain a large collection of facet-defining inequalities. The following example, taken from Cunningham [7], shows, however, that there are fractional extreme points in $(E F 1)$ and also $(E F 2)$.

For the above example, nodes 1,3 , and 5 are the terminal nodes. We have the following fractional extreme point:

$$
\begin{array}{r}
y^{i}(i)=1, y^{i}(i+1)=y^{i}(i-1)=z^{i}(i, i+1) \\
=z^{i}(i, i-1)=z^{i}(i-1, i+1)=\frac{1}{2}, i=3,5 ; \\
\begin{array}{r}
y^{1}(1)=1, y^{1}(2)=y^{1}(6)=z^{1}(1,2) \\
= \\
z^{1}(1,6)=z^{1}(6,2)=\frac{1}{2} ;
\end{array}
\end{array}
$$

and $y, z=0$ otherwise. By this choice of $y, z, x(e)=\frac{1}{2}$ for all edges in the graph. Assuming that $c(u, v)=2$ if the edge $(u, v)$ is incident to a terminal, $c(u, v)=1$ otherwise, then $Z_{E F 1}=Z_{E F 2} \leq 7.5$, while it can be easily seen the cost of any multiway cut is at least 8 , that is, $I Z_{M C} \geq 16 /$ $15 Z_{M C}$.

The computational experiments of Chopra and Owen [5] showed that the formulations $(E F 1)$ and $(E F 2)$ consistently yield high-quality bounds for the multiway cut problems. The previous example shows that the gap can be as large as $16 / 15$. We generalize the construction of this example to give an example whose gap is asymptotically close to 10/9.

Define the graph $G$ with nodes denoted $v_{1}, v_{2}, \ldots, v_{k}$ (terminals) and $u_{i, j}$, where $i \neq j, 1 \leq i, j \leq k$. Note that we assume $u_{i, j}$ and $u_{j, i}$ to be indistinguishable. The edge set of $G$ consists of edges $\left\{v_{i}, u_{i, j}\right\}$ for all $j$ and $\left\{u_{i, j}, u_{i, k}\right\}$ for all $k \neq j \neq i$. Let $c(u, v)=k-1$ if $(u, v)$ is incident to a terminal; otherwise, $c(u, v)=1$. When $k=3$, the construction reduces to the preceding example.

Let $y^{i}\left(u_{i, j}\right)=\frac{1}{2}, z^{i}\left(u_{i, j}, u_{i, k}\right)=\frac{1}{2}, z^{i}\left(u_{i, j}, v_{i}\right)=\frac{1}{2}$ for each $i=1, \ldots, k$. Thus, $x(u, v)=\frac{1}{2}$ for all edges in $G$. This yields a fractional $L P$ solution with cost

$$
Z_{E F 2}=\frac{k(k-1)^{2}}{2}+\frac{k(k-1)(k-2)}{4} .
$$

On the other hand, consider an optimum multiway cut solution. Let $T_{i}$ denote the set of nodes in the same component as the terminal $v_{i}$. Let $A_{i}$ denote the number of nodes in $T_{i}$ of the type $u_{i, j}$ for some $j, B_{i}=\left|T_{i}\right|-A_{i}$. Then, there are exactly $k(k-1)-\sum_{i=1}^{k} A_{i}$ edges with cost $k-1$ in the cut. Furthermore, there are at least $\left(k-2-A_{i}\right)\left(A_{i}\right)$ edges of the type $\left\{u_{i, j}, u_{i, k}\right\}$ in the cut. For each $u_{i, j}$, there are $(k-2)$ other neighbors of the type $u_{j, k}$. Hence, there are at least $(k-2) \sum_{i=1}^{k} A_{i}-2 \sum_{i=1}^{k} B_{i}$ edges of the type $\left\{u_{i, j}, u_{j, k}\right\}$ in the cut, the last term arising because there are at most $2 B_{i}$ edges between the nodes enumerated by $A_{i}$ and $B_{i}$ which do not belong to the cut. Note that each edge $\left\{u_{i, j}\right.$, $\left.u_{i, k}\right\}$ in the cut will be counted twice in this way as we vary over $i$. Hence, we have

$$
\begin{gathered}
I Z_{M C} \geq(k-1)\left(k(k-1)-\sum_{i=1}^{k} A_{i}\right)+\frac{1}{2}\left\{\sum_{i=1}^{k}\left(k-2-A_{i}\right)\right. \\
\left.\times\left(A_{i}\right)+(k-2) \sum_{i=1}^{k} A_{i}-2 \sum_{i=1}^{k} B_{i}\right\} \\
=(k-1) k(k-1)-\sum_{i=1}^{k} A_{i}-\sum_{i=1}^{k} \frac{A_{i}^{2}}{2}-\sum_{i=1}^{k} B_{i} .
\end{gathered}
$$

Since $\sum_{i=1}^{k} T_{i}=\sum_{i=1}^{k}\left(A_{i}+B_{i}\right)=k(k-1) / 2$, we have 


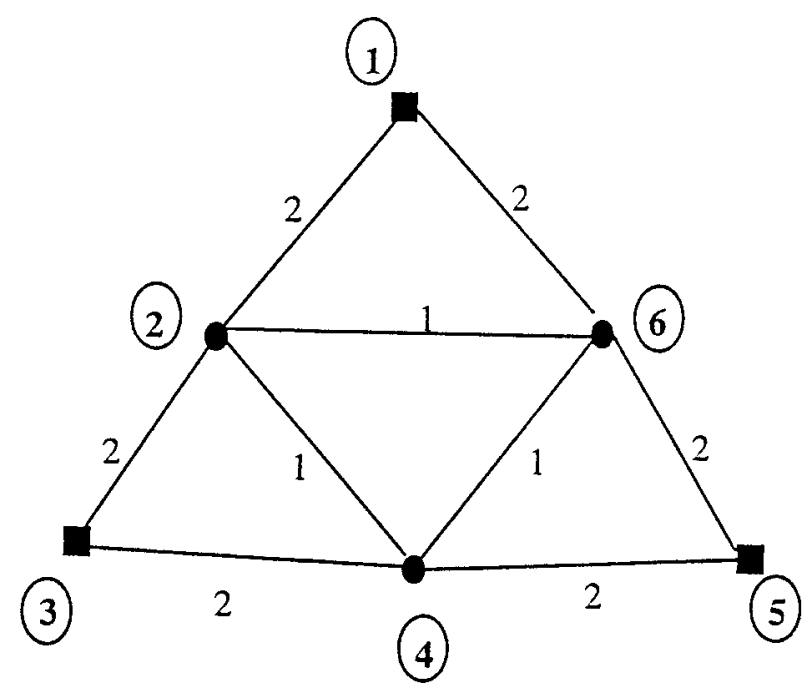

Fig. 1. An example with fractional extreme points.

$$
I Z_{M C} \geq k^{3}-\frac{\sum_{i} A_{i}^{2}}{2}+o\left(k^{3}\right) .
$$

It is easy to see that $\sum_{i} A_{i}^{2}$ is maximized when

- The node $u_{i, j}$ belongs to either $T_{i}$ or $T_{j}$.

- $A_{i} \neq A_{j}$ if $i \neq j$.

Hence,

$$
\sum_{i} A_{i}^{2} \leq 1^{2}+2^{2}+\cdots+(k-1)^{2}=\frac{k^{3}}{3}+o\left(k^{3}\right) .
$$

Using this bound, we have $I Z_{M C} \geq\left[\left(5 k^{3}\right) / 6\right]+o\left(k^{3}\right)$. Thus, $I Z_{M C} / Z_{E F 2} \geq 10 / 9$ asymptotically.

\subsection{Connection with Quadratic Zero-One Programming}

To strengthen the formulations further, we can consider stronger linearizations of the quadratic terms $y^{j}(u) y^{j}(v)$. The problem of linearizing quadratic terms of this type has been addressed within the context of unconstrained quadratic zero-one programming problems, leading to the Boolean Quadric polytope (BQP) (see Padberg [14] for a comprehensive treatment of the subject). The polyhedron $(B Q P)$ is also called the correlation polytope by some authors [12]. In this way, all valid inequalities known for the $B Q P$ can easily be converted to valid inequalities for the multiway cut problem. For instance, we can add the following valid inequalities:
- $z^{j}(u, u)+z^{j}(v, w) \geq z^{j}(u, v)+z^{j}(u, w)$,

- $z^{j}(u, u)+z^{j}(v, v)+z^{j}(w, w) \geq 1+z^{j}(u, v)+{ }^{j}(v, w)$ $+z^{j}(u, w)$.

Unfortunately, these valid inequalities do not cut off the fractional extreme point of Figure 1.

\section{RELATION BETWEEN THE MULTIWAY CUT AND THE INDEPENDENT SET PROBLEM}

In this section, we establish that the multiway cut problem on $G$ can be solved as an independent set problem on a related graph $I(G)$. We then use this connection to establish a new stronger extended formulation for the multiway cut problem.

We first describe the underlying intuition for our formulation. Consider a multiway cut solution $x$. For each terminal $v_{j}(j=1, \ldots, k)$, there is a set of nodes in $G$ which belongs to the same partition as terminal $v_{j}$. Let $E_{j}$ be the set of edges induced by these nodes (and terminal $v_{j}$ ). Clearly, the union of these edges (over $j$ ) is the complement of those edges in the multiway cut solution. We attach a label to these edges, with label $j$ assigned to edges in the set $E_{j}$. We will construct a new graph $I(G)$ such that nodes in this graph correspond to the edges (with a label attached) in $G$. Furthermore, using edges in a multiway cut solution, and by selecting the labels appropriately, we can obtain a corresponding independent set in $I(G)$.

Formally, given a graph $G$, let $I(G)$ denote the graph with node set

$$
\begin{aligned}
& \{(u, v, j):(u, v) \in E(G), j \\
& \quad=1,2, \ldots, k\} \backslash\left\{\left(v_{j}, u, i\right): i \neq j,\left(v_{j}, u\right) \in E(G)\right\} .
\end{aligned}
$$

Note that nodes of the type $\left(v_{j}, u, i\right)$ with $i \neq j$ do not exist in $I(G)$ since the edge $\left(v_{j}, u\right)$ will never be in the same partition as terminal $v_{i}$. Let the edge set of $I(G)$ consist of edges of the type

$$
\begin{aligned}
\left\{\left(\left(u_{1}, w_{1}, i\right),\left(u_{2}, w_{2}, j\right)\right):\right. & \\
& \left.i \neq j ;\left|\left\{u_{1}, w_{1}\right\} \cap\left\{u_{2}, w_{2}\right\}\right| \geq 1\right\} .
\end{aligned}
$$

Thus, two nodes in $I(G)$ are adjacent if the corresponding edges in $G$ are adjacent, unless the label attached to the edges (in $G$ ) are identical.

Consider a maximal independent (stable) set $I$ in $I(G)$. Let $F_{j}=\{(u, v) \in E(G):(u, v, j) \in I\}$. The edgeinduced subgraphs $G\left[F_{j}\right]$ are node disjoint in the graph $G$, since $I$ is a stable set. Moreover, by maximality of $I$, each nonterminal node must be a node in one of the subgraphs $G\left[F_{j}\right]$. This partition induces a solution to the multiway cut 
problem and vice versa. For each node $(u, v, j)$ in $I(G)$, we assign costs $c(u, v)$. Then, the cost of the multiway cut in $G$ and the cost of the maximum independent set in $I(G)$ are related as follows:

$I Z_{M C}=\sum_{(u, v) \in E(G)} c(u, v)-\max \{c(I): I$ stable set in $I(G)\}$.

The problem of finding a maximum weight stable set in $I(G)$ can be formulated as

$($ IREF $) \quad Z_{\text {IREF }}=\max \sum_{(u, v) \in E} \sum_{j=1}^{k} c(u, v) z^{j}(u, v)$

subject to $\quad z^{i}(u, v)+z^{j}(u, v) \leq 1$,

$$
\begin{array}{r}
i \neq j,(u, v) \in E \\
z^{i}(u, v)+z^{j}(u, w) \leq 1, \quad i \neq j,(u, v),(u, w) \in E \\
z^{j}(u, v) \in\{0,1\}, \quad \forall j,(u, v) \in E .
\end{array}
$$

Let $(R E F)$ denote the linear relaxation of (IREF).

This correspondence has many interesting consequences: As an example, since the multiway cut for fixed $k \geq 3$ is NP-hard, we obtain as a direct corollary that the maximum independent set problem on a $k$-partite graph $(k \geq 3)$ is also NP-hard.* In addition, many classes of facet-defining inequalities for the stable set problem can be interpreted (via proper translation) as valid inequalities for the multiway cut problem.

For instance, a natural way to strengthen $(R E F)$ is to include the maximal clique inequalities (cf. [2]) for the stable set problem. The maximal cliques in $I(G)$ are of the form

$$
\left\{\left(u, w_{1}, 1\right), \ldots,\left(u, w_{k}, k\right)\right\}
$$

where $\left(u, w_{i}\right) \in E(G)$ or

$$
\left\{\left(e_{1}, 1\right), \ldots,\left(e_{k}, k\right)\right\},
$$

where $e_{i} \in\{(u, w),(u, v),(v, w)\}$ are edges of a triangle in $G$.

Putting all these together, we have the following valid relaxation for the multiway cut problem:

$(I N D)$

$$
Z_{I N D}=\min \sum_{(u, v) \in E} c(u, v)\left(1-\sum_{j} z^{j}(u, v)\right)
$$

\footnotetext{
* We believe that this result is known but we have not been able to locate any reference in the literature.
}

subject to

$$
\sum_{j} z^{j}\left(u, w_{j}\right) \leq 1
$$

$\forall u$, with $w_{j}$ adjacent to $u$

$$
\begin{gathered}
\sum_{j} z^{j}\left(e_{j}\right) \leq 1 ; \quad e_{j} \text { edges of a common triangle } \\
0 \leq z^{j}(u, v) \leq 1, \quad \forall j,(u, v) \in E .
\end{gathered}
$$

It is not difficult to see that (IND) is at least as strong as (EF2) [and, hence, $(E F 1, E F 3)]$.

Theorem 5. If $G$ is triangle free, then $Z_{I N D}=Z_{E F 2}$; else, $Z_{I N D} \geq Z_{E F 2}$.

Proof. Let $(z)$ be an optimal solution to $(I N D)$. Let $z^{j}(e)$ $=0$ if $e$ is incident to a terminal $v_{l}$ with $l \neq j$. Let $y^{j}(u)$ $=\max _{e} z^{j}(e)$ where the maximum is taken over all edges $e$ incident to $u$ in $G$, and $(e, j) \in V(I(G))$. Let $y^{j}\left(v_{l}\right)=0$ if $l \neq j$. Then, the solution $(z, y)$ satisfies

$$
\begin{gathered}
\sum_{j=1}^{k} z^{j}(u, v) \leq 1 \forall(u, v) \in E ; \\
z^{j}(u, v) \leq \min \left(y^{j}(u), y^{j}(v)\right) \forall(u, v) \in E ; \\
\sum_{j=1}^{k} y^{j}(u) \leq 1 \forall u \in V \backslash T ; \\
y^{j}\left(v_{j}\right) \leq 1 \forall j=1, \ldots, k .
\end{gathered}
$$

In $(E F 2)$, we can increase the value of $y$ to ensure equality at constraints (1)-(3). In this way, the modified $(z, y)$ is clearly feasible in $(E F 2)$ with the same objective value as $Z_{I N D}$. Hence,

$$
Z_{E F 2} \leq Z_{I N D}
$$

On the other hand, if $G$ does not contain any triangles, the constraints in $(E F 2)$ imply that

$$
\sum_{j=1}^{k} z^{j}\left(u, w_{j}\right) \leq \sum_{j=1}^{k} y^{j}(u)=1 .
$$

Hence, $Z_{I N D} \leq Z_{E F 2}$.

Consider the example as shown in Figure 1. Recall that

$$
\begin{aligned}
y^{i}(i) & =1, y^{i}(i+1)=y^{i}(i-1)=z^{1}(i, i+1) \\
& =z^{1}(i, i-1)=z^{1}(i-1, i+1)=\frac{1}{2}, \quad i=1,3,5 .
\end{aligned}
$$


This fractional optimal solution violates the triangle inequality

$$
z^{1}(2,6)+z^{3}(2,4)+z^{5}(4,6) \leq 1 .
$$

Hence, $(I N D)$ can be strictly stronger than those formulations considered in the previous section.

In general, the clique constraints for the independent set problem cannot be separated in polynomial time (unless $P$ $=N P)$. In this case, because of the specific nature of $I(G)$, we can separate the clique constraints in $I(G)$ in polynomial time. Hence, $(I N D)$ is solvable in polynomial time via the ellipsoid algorithm.

Theorem 6. (IND) is solvable in polynomial time.

Proof. For each node $u$ in $G$, the inequalities

$$
\sum_{j} z^{j}\left(u, w_{j}\right) \leq 1, \forall w_{j} \text { adjacent to } u,
$$

are satisfied if and only if the inequality is satisfied by a single choice of $w_{j}$. In particular, choose

$$
w_{j}=\operatorname{argmax}\left\{z^{j}(u, w): w \text { adjacent to } u\right\} .
$$

Similarly, the inequalities corresponding to the triangles can be checked by verifying only for the case $e_{j}=\operatorname{argmax}\left\{z^{j}(e)\right.$, $\left.z^{j}(f), z^{j}(g)\right\}$. that

It follows directly from the theory of perfect graphs ([2])

Theorem 7. If $I(G)$ is a perfect graph, formulation (IND) for the multiway cut problem is integral.

Note that for $k=2$ (i.e., for the $s-t$ cut problem) $I(G)$ is bipartite and is therefore a perfect graph. Thus, (IND) is always integral in this case.

Other valid inequalities for the multiway cut problem can be constructed from facets for the independent set problem. For example, the well-known odd-cycle inequalities for the stable set problem translate to

$$
\sum_{i=1}^{l} z^{j(i)}\left(e_{i}\right) \leq\lfloor|C| / 2\rfloor,
$$

where $C=\left\{\left(e_{i}, j(i)\right)\right\}_{i=1}^{l}$ is an odd cycle in $I(G)$. It is well known (see [11]) that the odd-cycle inequalities can be separated in polynomial time.

\subsection{Polynomially Solvable Cases of the Multiway Cut Problem}

Chopra and Owen [5] showed that (EF2) is integral when the underlying graph $G$ is a tree. We show that this result is a direct corollary of Theorem 7 . We will need some definitions and terminology from graph theory. Let $G$ and $H$ be two distinct graphs, each containing a clique of size $k$. Let $K_{1}$ and $K_{2}$ be cliques of size $k$ in $G$ and $H$, respectively. By gluing $G$ and $H$ together on a $k$-clique, we obtain a new graph $L$ with $V(L)=\left(V(G) \backslash V\left(K_{1}\right)\right) \cup\left(V(H) \backslash V\left(K_{2}\right)\right)$ $\cup V(K)$, where $K$ is a clique of size $k$, and $\phi_{1}: V\left(K_{1}\right) \rightarrow V(K)$ and $\phi_{2}: V\left(K_{2}\right) \rightarrow V(K)$ are 1-1 maps, with $e=(u, v) \in E(L)$ if and only if

$$
u, v \notin V(K), \quad(u, v) \in E(G) \cup E(H), \quad \text { or }
$$

$$
u, v \text { both in } V(K), \text { or }
$$

$$
\begin{array}{ll}
v \in V(K), \quad u \in V(G) \backslash V(K), \quad\left(u, \phi_{1}^{-1}(v)\right) \in E(G), \quad \text { or } \\
v \in V(K), \quad u \in V(H) \backslash V(K), \quad\left(u, \phi_{2}^{-1}(v)\right) \in E(H) .
\end{array}
$$

Theorem 8 [5]. Formulation (EF2) is integral when $G$ is a tree.

Proof. Since $G$ does not contain any triangles, $(E F 2)$ coincides with the formulation (IND). It suffices, then, to show that $I(G)$ is perfect. If $G$ corresponds to a star on $n$ +1 nodes, then $I(G)$ is a complete $n$-partite graph and therefore perfect. Since all trees $G$ are formed by "gluing" star graphs on cut-edges, $I(G)$ is formed by clique-gluing of complete multipartite graphs. Since clique-gluing operations preserve perfection (cf. [2]), $I(G)$ is perfect, when $G$ is a tree. Therefore, $(I N D)$ and, hence, $(E F 2)$ is tight in this instance.

This result is interesting, as the multiway cut problem over trees has an important generalization which arises in biomathematics. The generalized multiway cut problem introduced in Erdos and Székely [9] is as follows: Given a graph $G=(V, E)$ and a partial $k$-coloring of the nodes, that is, a subset $V^{\prime} \subseteq V$ and a function $f: V^{\prime} \rightarrow\{1, \ldots, k\}$, find an extension of $f$ to $V$ such that the total weight of edges with different colored endpoints is minimized. Erdos and Székely [9] contains a nice illustration of how this problem arises naturally in the study of evolutionary trees. They also constructed a polynomial time dynamic programming algorithm for the generalized multiway cut problem on trees. In $(E F 2)$, this amounts to setting

$$
y^{f(u)}(u)=1, \quad y^{j}(u)=0 \quad \text { otherwise }
$$

for each $u$ in $V^{\prime}$. Since faces of an integral polytope are also integral, the generalized multiway cut problem is also 


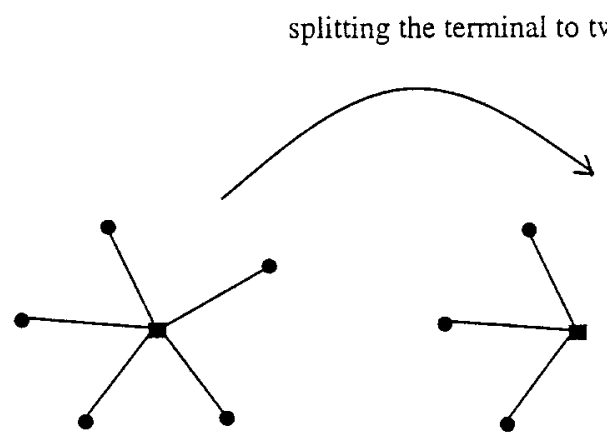

- Terminal node

Fig. 2. Splitting of terminals.

solved by the modified version of ( $E F 2)$ when $G$ is a tree. Using this notion of a generalized multiway cut, it follows directly (see Erdos and Székely [9]) that if the set of terminals $V(H)$ in $G$ intersects every cycle in $G$ then by splitting the terminals into multiple terminals with the same colors (see Fig. 2 for the splitting operation) we can transform the multiway cut problem on $G$ to a generalized multiway cut problem on a forest. Hence, the multiway cut problem is solvable in polynomial time if the terminals intersect every cycle in $G$.

In the rest of this section, we exploit the stronger formulation (IND) to improve on these results.

Let $G$ be a graph that can be obtained via node gluing of triangles and edges (see Fig. 3 for an example). We call such graph a triangular cactus.

Lemma 9. If $G$ is a triangular cactus, $I(G)$ is perfect.

Proof. We only sketch the main idea of the proof. We proceed by induction, using the fact that $G$ is built up by node gluing of edges and triangles. The case when $G$ is a triangle or an edge is trivial. Suppose now that $G$ is formed by node gluing of 2 triangular cacti $G_{1}$ and $G_{2}$ at the node $v$. Let $M$ be a node-induced subgraph of $I(G)$. Let $H(M)$ be the corresponding set of labeled edges in $G$. Let $q(M)$ denote the size of a maximum clique in $M$. We only need to show that the chromatic number of $M$ is at most $q(M)$. All the labeled edges in $H(M)$ that are incident to $v$ induce a complete multipartite subgraph in $I(G)$ which can be colored using at most $q(M)$ colors. With these nodes colored, the problem reduces to that of two smaller coloring problems on $M \cap I\left(G_{1}\right)$ and $M \cap I\left(G_{2}\right)$. It follows from the induction hypothesis that each of these two problems can be colored using at most $q(M)$ colors.
Theorem 10. If the set of terminals $T$ in $G$ intersects every cycle of length greater or equal to 4 , then the multiway cut problem is solvable in polynomial time.

Proof. By splitting terminals as in Erdos and Székely [9] (see also Fig. 2), the multiway cut problem in the above theorem can be transformed to a generalized multiway cut problem on triangular cactus. Hence, the theorem follows immediately from Lemma 9. For details, we refer the readers to the original proof in [9].

\section{TIGHTNESS OF THE LP RELAXATIONS}

In this section, we analyze the tightness of the various LP relaxations proposed in the previous sections. Note that the technique of Cunningham [7] implies a bound of 2 for these formulations, but his analysis uses the result of Lovász's multiflow theorem. On the other hand, Dalhaus et al. [8] proposed a 2-approximation algorithm for the multiway cut problem. We show that the technique of Dalhaus et al. can be suitably extended to analyze the LP relaxations obtained above. This is achieved via a suitable randomized rounding extension of their heuristic. The rounding approach, coupled with better combinatorial approximation algorithms for the 4- and 8-terminal cut problems, led naturally to new and tighter LP relaxations for the corresponding problems.

\section{Randomized Rounding Heuristic H.}

1. Solve the relaxation (EF3), obtaining an optimal solution $(\bar{x}, \bar{y})$.

2. Generate a random number $U$, uniformly between 0 and 1 .

3. For each node $u$ and $j$, set $y^{j}(u)$ to 1 if $U \leq \bar{y}^{j}(u)$ and 0 otherwise. In this way, sets $S_{j}$ (not necessarily disjoint) are generated, $j=1, \ldots, k$, such that $S_{j}$ contains terminal node $v_{j}$ but not $v_{i}$ for $i \neq j$ [recall that $y^{j}\left(v_{i}\right)$ $=0$ if $i \neq j$ ]. Compute $c\left(\delta\left(S_{j}\right)\right)$. Let $S_{\text {max }}=\operatorname{argmax}$ $c\left(\delta\left(S_{j}\right)\right)$.

4. The proposed solution is the set of edges in

$$
D=\cup_{j: S_{j} \neq S_{\max }} \delta\left(S_{j}\right) .
$$
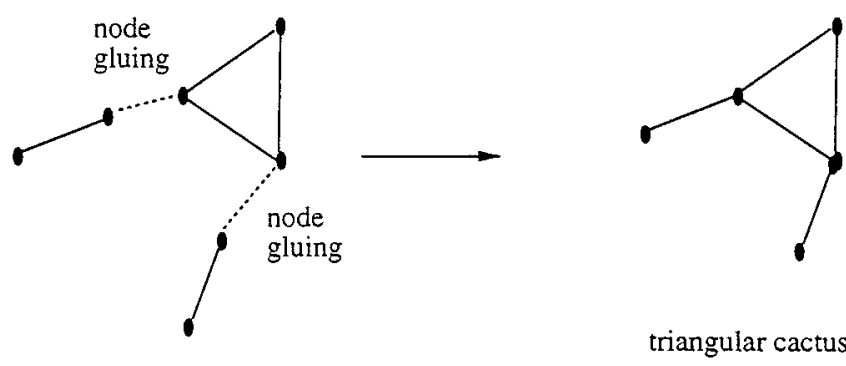

Fig. 3. Triangular cactus. 
Clearly, the solution is feasible for the multiway cut problem. Let $Z_{H}$ be the value of the heuristic.

Theorem 11. For $c \geq 0, I Z_{M C} \leq E\left[Z_{H}\right] \leq 2(1-1 / k) Z_{E F 3}$.

Proof. Clearly, the value of the multicut $D$ is

$$
c(D) \leq(1-1 / k) \sum_{j} c\left(\delta\left(S_{j}\right)\right) .
$$

Since

$$
\begin{aligned}
P\left\{(u, v) \in \delta\left(S_{j}\right)\right\} & =P\left\{( y ^ { j } ( u ) = 1 , y ^ { j } ( v ) = 0 ) \cup \left(y^{j}(u)\right.\right. \\
=0, y^{j}(v)= & 1)\}=P\left\{\min \left(\bar{y}^{j}(u), \bar{y}^{j}(v)\right) \leq U\right. \\
& \left.\leq \max \left(\bar{y}^{j}(u), \bar{y}^{j}(v)\right)\right\}=\left|\bar{y}^{j}(u)-\bar{y}^{j}(v)\right|,
\end{aligned}
$$

we have

$$
\begin{aligned}
E\left[\sum_{j} c\left(\delta\left(S_{j}\right)\right)\right]=\sum_{j=1}^{k} \sum_{(u, v) \in E} c(u, v) P\left\{(u, v) \in \delta\left(S_{j}\right)\right\} \\
\quad=\sum_{j=1}^{k} \sum_{(u, v) \in E} c(u, v)\left|\bar{y}^{j}(u)-\bar{y}^{j}(v)\right| .
\end{aligned}
$$

From $(E F 3)$, we know that

$$
\sum_{j=1}^{k}\left|\bar{y}^{j}(u)-\bar{y}^{j}(v)\right| \leq 2 \bar{x}(u, v) .
$$

Hence,

$$
E\left[\sum_{j} c\left(\delta\left(S_{j}\right)\right)\right] \leq 2 \sum_{(u, v) \in E} c(u, v) \bar{x}(u, v) .
$$

Therefore,

$$
\begin{aligned}
E\left[Z_{H}\right]=E[c(D)] \leq(1-1 / k) E\left[\sum_{j} c\left(\delta\left(S_{j}\right)\right)\right] & \\
& \leq 2(1-1 / k) Z_{E F 3} .
\end{aligned}
$$

Using the conditioning method (see Alon and Spencer [1]), we can make Step 2 of the randomized heuristic deterministic. So, we obtained an approximation algorithm that delivers a multiway cut at most twice the optimal. Another easier way to obtain a deterministic 2-approximation algorithm is to find, for each $j$, a minimum cut containing the terminal $v_{j}$ but not the other terminals, this time among the sets $\left\{v_{j}, u_{1}, u_{2}, u_{3}, \ldots\right\}$ with the nodes ordered (in nonincreasing order, breaking ties arbitrarily) according to the value of $y^{j}\left(u_{i}\right)$. Dalhaus et al. [8] constructed directly a combinatorial algorithm to approximate the multiway cut problem, also within a bound of $2(1-1 / k)$. However, our result is a little stronger in that the bound on the heuristic solution is in terms of the linear programming relaxation and not the optimal integer solution.

\subsection{The Case $k=4$}

Dalhaus et al. [8] showed that the approximation bound $\frac{3}{2}$ can be improved further to $\frac{4}{3}$ for the 4-terminal cut problems. By a randomized version of their heuristic, we propose next a linear relaxation attaining the same worst-case bound of $\frac{4}{3}$ for the 4-terminal cut problem:

$$
Z_{4 T}=\min \sum_{(u, v) \in E} c(u, v) x(u, v)
$$

$$
\begin{array}{r}
\text { subject to } 2 x(u, v) \geq \sum_{i=2}^{4}\left|y^{1}(u)+y^{i}(u)-y^{1}(v)-y^{i}(v)\right| ; \\
\forall(u, v) \in E
\end{array}
$$

and constraints (1)-(4). Note that the above relaxation is valid only for the case $k=4$. In this regard, it is less general than the formulation in $(E F 3)$. Furthermore, this convex programming problem is essentially a linear program. We keep this form as it makes the following analysis more transparent. Let $I Z_{4 T}$ be the corresponding optimal integer programming value.

Theorem 12. $I Z_{4 T} \leq \frac{4}{3} Z_{4 T}$.

Proof. Let $(x, y)$ be an optimal solution to $Z_{4 T}$. We generate randomly cuts of the form $F(1, i)$, which separates terminals $v_{1}, v_{i}$ from the other two terminals, in the following way:

- $F(1, i)=\varnothing$. Generate $U$ randomly on $[0,1]$.

- If $y^{1}(u)+y^{i}(u) \geq U$, then $F(1, i) \leftarrow F(1, i) \cup\{u\}$. Repeat for all $u$.

Note that the edge $(u, v)$ is in the cutset $F(1, i)$ only when

$$
\begin{aligned}
& y^{1}(u)+y^{i}(u) \geq U, y^{1}(v)+y^{i}(v)<U ; \text { or } \\
& y^{1}(v)+y^{i}(v) \geq U, y^{1}(u)+y^{i}(u)<U .
\end{aligned}
$$

Let $\delta(S)$ denote the set of edges in the cut set $S$ :

$$
\begin{aligned}
E[c(\delta(F(1, i)))]= & \sum_{(u, v)} c(u, v) \\
& \quad \times\left|y^{1}(u)+y^{i}(u)-y^{1}(v)-y^{i}(v)\right| .
\end{aligned}
$$

Now, since the union of any two of the three cuts generated 
is a valid 4-terminal cut, by taking the minimum $Z_{H}$ of the three feasible solutions, we have

$$
Z_{H} \leq \frac{2}{3} \sum_{i=2}^{4} E\left(c(\delta(F(1, i))) \leq \frac{4}{3} Z_{4 T}\right.
$$

Remark. Similarly, the combinatorial approximation algorithm for the 8-way-cut problem [8] can be turned into an LP formulation with equivalent bound to the 8-way-cut problem.

\section{THE MULTICUT PROBLEM}

The results in the previous section have natural extensions to the more general multicut problem. The technique of Dalhaus et al. [8] can be extended to yield a $2 \alpha(H)(1$ $-1 / k)$ approximation algorithm for the multicut problem, where $\alpha(H)$ denotes the size of the maximum independent set in the demand graph $H$. In contrast, Garg et al. [10] designed an $O(\log |V(H)|)$ approximation algorithm for this problem. Neither bound dominates the other. The former can be much better in a dense graph, as in the case of the multiway cut problem, where $H$ is a clique. The bound obtained by Garg et al. [10] is via a natural linear relaxation of the multicut problem, based on a multicommodity flow formulation. In this section, we introduce a different linear formulation for the multicut problem, which can be viewed as the LP counterpart for the extension of Dalhaus et al.'s combinatorial algorithm:

$$
Z_{M 2}=\min \sum_{(u, v) \in E} c(u, v) x(u, v)
$$

$$
\begin{aligned}
\text { subject to } \quad 2 \alpha(H) x(u, v)+\sum_{j} z^{j}(u, v) \geq \sum_{j \in S} y^{j}(u) \\
+\sum_{j \notin S} y^{j}(v) ; \quad(u, v) \in E, S \subset V(H) \\
z^{j}(u, v) \leq y^{j}(u), \quad \forall j,(u, v) \in E \\
z^{j}(u, v) \leq y^{j}(v), \quad \forall j,(u, v) \in E \\
\sum y^{j}(u) \leq \alpha(H), \quad \forall u \in V \\
y^{j}\left(s_{j}\right)=1 ; \quad \forall j \\
y^{j}(v)=0 ; \quad \text { if }\left(v, s_{j}\right) \in E(H) \\
0 \leq x(u, v), y^{j}(u) \leq 1, \quad \forall j,(u, v) \in E .
\end{aligned}
$$

Let $I Z_{M}$ denote the value of an optimal multicut solution.
Theorem 13. $Z_{M 2} \leq I Z_{M} . Z_{M 2}$ can be computed in polynomial time.

Proof. Consider the incidence vector $x$ of any multicut solution. Let $y^{j}(u)=1$ if node $u$ and $v_{j} \in V(H)$ lie in the same component in the multicut. Let $z^{j}(u, v)=y^{j}(u) y^{j}(v)$. If $u, v$ lie in the same component $C$, then

$$
\sum_{j \in S} y^{j}(u)+\sum_{j \notin S} y^{j}(v)=|V(H) \cap C|=\sum_{j \in V(H)} z^{j}(u, v) .
$$

If $u, v$ lie in two different components $C_{1}$ and $C_{2}$, then $\forall$ $S \subset V(H)$ :

$$
\sum_{j \in S} y^{j}(u)+\sum_{j \notin S} y^{j}(v) \leq 2 \alpha(H)
$$

Hence,

$$
2 \alpha(H) x(u, v)+\sum_{j} z^{j}(u, v) \geq \sum_{j \in S} y^{j}(u)+\sum_{j \notin S} y^{j}(v)
$$

is a valid inequality. Clearly, $\sum_{j} y^{j}(u) \leq \alpha(H)$ is also a valid inequality. Since

$$
\max \left\{\sum_{j \in S} y^{j}(u)+\sum_{j \notin S} y^{j}(v): S \subset A\right\}
$$

is solvable in polynomial time (see [5]), we can solve (M2) in polynomial time by the ellipsoid method.

We next apply the same randomized rounding heuristic $H$ to the optimal solution of (M2):

Theorem 14. $I Z_{M} \leq E\left[Z_{H}\right] \leq 2 \alpha(H)\left(1-\frac{1}{|V(H)|}\right) Z_{M 2}$.

Proof. Let $(\bar{x}, \bar{y}, \bar{z})$ be an optimal solution to $(M 2)$. Then,

$$
\begin{aligned}
E\left[\sum_{j} c\left(\delta\left(S_{j}\right)\right)\right]= & \sum_{j} \sum_{(u, v) \in E(G)} c(u, v)\left|\bar{y}^{j}(u)-\bar{y}^{j}(v)\right| \\
\leq & \sum_{(u, v) \in E(G)} c(u, v)\left\{\sum_{j \in T(u, v)}\left(\bar{y}^{j}(u)-\bar{z}^{j}(u, v)\right)\right. \\
& +\sum_{j \notin T(u, v)}\left(\bar{y}^{j}(v)-\bar{z}^{j}(u, v)\right) \\
\leq & 2 \alpha(H) \sum_{(u, v) \in E(G)} c(u, v) \bar{x}(u, v) \\
& =2 \alpha(H) Z_{M 2} .
\end{aligned}
$$


By discarding the set $S_{\max }$ with the maximum cut, we obtain a multicut solution $D^{\prime}$ such that

$$
\begin{aligned}
& E\left[Z_{H}\right]=E\left[c\left(D^{\prime}\right)\right] \leq(1-1 / k) E\left[\sum_{j} c\left(\delta\left(S_{j}\right)\right)\right] \\
& \leq 2 \alpha(H)\left(1-\frac{1}{|V(H)|}\right) Z_{M 2} .
\end{aligned}
$$

\section{CONCLUDING REMARKS}

In this paper, we proposed several new reformulations for multiway cut and multicut problems. We showed that these formulations are derived from a tight nonlinear formulation. By reducing the multiway cut problem to the maximum independent set problem, we also bring the tools of perfect graph theory to bear on several solvable instances of the multiway cut problems. Furthermore, we show that the well-known Dalhaus et al. algorithm can be used in a randomized rounding framework to analyze the worst-case behavior of these formulations. Several problems are left open in the paper; for instance, we have not been able to close the gap between the upper-bound 2 and the worst-case example which attains a bound of 10/9 asymptotically. Furthermore, we have not been able to analyze whether the stronger formulation (IND) yields a better bound in this case.

The research of one of the authors (D.B.) was partially supported by a Presidential Young Investigator Award DDM9158118

\section{REFERENCES}

[1] N. Alon and J.H. Spencer, The probabilistic method, Wiley, New York, 1992.
[2] C. Berge and V. Chvátal (Editors), Topics on perfect graphs, Anal Discr Math 21 (1984).

[3] D. Bertsimas, C.P. Teo, and R. Vohra, "On dependent randomized rounding algorithms," 5th Integer Programming and Combinatorial Optimization Conf, W. Cunningham, S. McCormick, and M. Queyranne (Editors), LNCS 1084, Springer-Verlag, Berlin, 1996, pp. 330-344.

[4] B.V. Cherkasskij, Solution of a problem on multi commodity flows in a network, Ehkon Mat Metody 13 (1977), 143-151.

[5] S. Chopra and L.H. Owen, Extended formulations for the A-cut problem, Math Program (1996), preprint.

[6] S. Chopra and M.R. Rao, On the multiway cut polyhedron, Networks 21 (1991), 51-89.

[7] W.H. Cunningham, The optimal multi terminal cut problem, DIMACS Ser Discr Math Theor Comput Sci 5 (1991), 105-120.

[8] E. Dalhaus, D. Johnson, C. Papadimitriou, P. Seymour, and M. Yannakakis, Complexity of multi terminal cuts, SIAM J Comput 23 (1990), 864-894.

[9] P. Erdos and L.A. Székely, On weighted multiway cuts in trees, Math Program 65 (1994), 93-105.

[10] N. Garg, V. Vazirani, and M. Yannakakis, Approximate max-flow min-(multi)cut theorems and their applications, SIAM J Comput 25 (1996), 235-251.

[11] M. Grötschel, L. Lovász, and A. Schrijver, Geometric algorithms and combinatorial optimization, Springer-Verlag, Berlin, 1988.

[12] M. Laurent and S. Poljak, On the facial structure of the set of correlation matrices, Technical report, 1994.

[13] L. Lovász, On some connectivity properties of Eulerian graphs, Acta Math Akad Sci Hung 28 (1976), 129-138.

[14] M. Padberg, The Boolean quadric polytope: Some characteristics, facets and relatives, Math Program 45 (1989), 139-172.

[15] C.P. Teo and D. Bertsimas, "On improved randomized algorithms for lotsizing problems," 5th Integer Programming and Combinatorial Optimization Conf, W. Cunningham, S. McCormick, and M. Queyranne (Editors), LNCS 1084, Springer-Verlag, Berlin, 1996, pp. 359-373. 